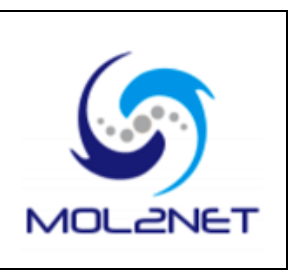

\title{
Electrical study of new anthracene derivatives for electronics applications
}

\author{
Ghada Attia*, Safa Teka, Mustaphe Majdoub, Rafik Ben Chaabane
}

Laboratoire des Interfaces et des Matériaux Avancés (LIMA), Université de Monastir Faculté des Sciences de Monastir, Bd. de l'Environnement, 5019 Monastir, Tunisia. (S T.) safateka@gmail.com; (M.M.) mustapha.majdoub@fsm.rnu.tn; (R.B.C) rafik.benchaabane@ fsm.rnu.tn

*Author to whom correspondence should be addressed; Attia-Ghada@hotmail.com, Tel.: $+33758846473$

Received: / Accepted: / Published:

\begin{abstract}
Due to the holes high transport mobility, anthracene derivates are widely used for the elaboration of OLEDs and other organic thin-layer-based electronic, such as transistors and photovoltaic cells. This study aims to investigate the electric properties of two new anthracene derivatives: 9-\{[4(\{[4-(9 anthrylmethoxy)phenyl]sulfanyl $\}$ methyl)] methyl $]$ anthracene (TDP-AN) and 4-(9anthrylmethoxy) benzyl[4-(9-anthrylmethoxy)phenyl]sulfone (BPS-AN). The main difference between them is related to the functional group between the two central phenyls. Conductance measurements results show a frequency-independent behaviour, for low frequencies ranging between 0.1 and $100 \mathrm{KHz}$, while they exhibit a power law model for higher ones. BPS-AN was found more conductive than the TDP-AN. Impedance spectroscopy (IS) was then investigated to understand the charge carrier mechanisms in both TDP-AN and BPS-AN molecules. Measurements were carried out in the frequency range $100 \mathrm{~Hz}-10 \mathrm{MHz}$ at different bias voltages (from $0 \mathrm{~V}$ to $3 \mathrm{~V}$ ). The obtained results agree with conductivity measurement ones.
\end{abstract}

Keywords: Anthracene derivates; Conductance, Impedance spectroscopy, Charge carrier.

\section{Introduction}

Organic semiconducting materials are very attractive for electronic applications such as organic thin-film transistors (OTFTs), organic photovoltaic cells $[1,2]$ and organic light-emitting diodes (OLEDs).

Several organic monolayers have been investigated for electronics applications. Our work concerns anthracene derivatives because of their multiple advantages, such as improved electrical conduction making them good materials for elaboration of organic diodes.
In this study we have chosen to investigate the electric properties of two new anthracene derivatives: $\quad 9-\{[4-(\{[4-(9$ anthrylmethoxy)phenyl]sulfanyl \}methyl)]methy] anthracene(TDP-AN) and 4-(9-anthrylmethoxy) benzyl[4-(9-anthrylmethoxy)phenyl]sulfone (BPS-AN). 


\section{Results and Discussion}

For conductance and impedance measurements, each anthracene derivative was placed between an aluminum $(\mathrm{Al})$ cathode and a tin oxide (ITO) anode (Figure. 1). The realized structures were then characterized by impedance spectroscopy, a powerful technique for exploring the charge carrier mechanisms in organic semiconductors.

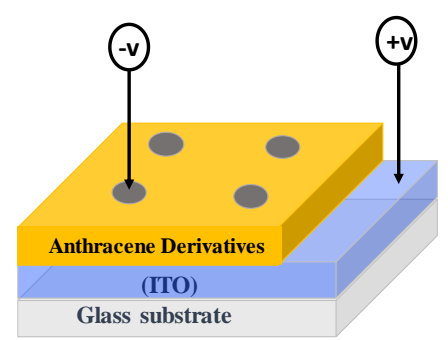

Figure. 1. [ITO/ anthracene derivative/Al] diode structure

\subsection{The conductance measurements:}

The variation of conductance versus frequency at different bias voltages for the two realized devices is presented in Figures. $2 a$ and $2 b$. These spectra present the steps performed in the frequency range $100 \mathrm{~Hz}-10 \mathrm{MHz}$. Results indicate the presence of two parts, a quasiconstant conductance at low frequencies and an increase, according a power-law, for high ones. Besides, the conductance increases with the applied bias voltage.

In general, the variation of conductance $G$ according to frequency in disordered materials obeys the relation:
The dc conductance $\left(G_{d c}\right)$ remains constant at low frequencies $(0.1-100 \mathrm{kHz})$. Thus, a charge carrier jumps from one position to its neighbouring vacant one [3]. Beyond a critical frequency of order of $100 \mathrm{kHz}$, the conductance starts to increase. Consequently, a charge carrier will hop from a site to another one [3].

According to the conductance at $0 \mathrm{~V}$, obtained in Results gathered in Figure. 2c indicate that BPS-AN is more conductive than TDP-AN.

\subsection{Parameters obtained by modeling the impedance spectra}

Cole-Cole representation for both devices at different bias voltages are shown in Figures.3a. and $3 \mathrm{~b}$. The application of a voltage to the device, results in a single semicircle whose radius decreases with increasing the bias from $0 \mathrm{~V}$ to $3 \mathrm{~V}$. The minimum value of $\operatorname{Re}(\mathrm{Z})$ (high frequency) indicates the existence of voltage-independent series resistance $R_{s}$ which corresponds to the ohmic contact with the interface holes-injecting ITO/organic material. The maximum value of $\mathrm{Re}$ (Z) (low frequency) represents the sum of $R_{p}$ parallel resistance with $R_{S}$ contact resistance. For the both structures, the bulk resistance $R_{p}$ decreases with increasing bias.

The obtained values, gathered in Figure $3 \mathrm{c}$., indicate that ITO/BPS-AN/Al structure has a lower resistance than that of ITO/TDP-AN/Al one, in concordance with the conductivity measurement results.

$$
G(\omega)=G_{d c}+G_{a c}(\omega)
$$
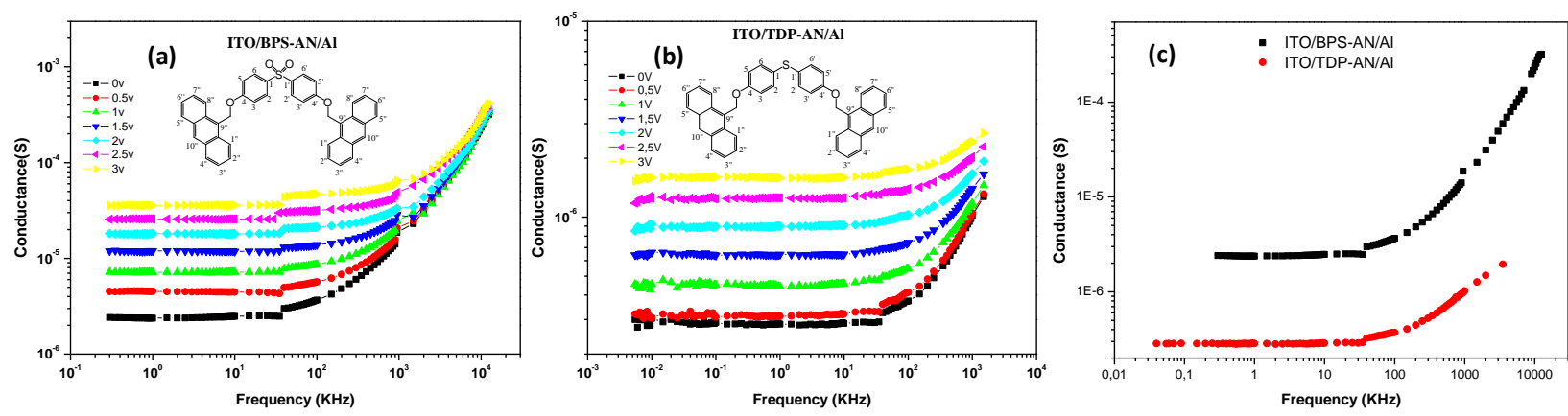

Figure 2. Conductance versus frequency at different applied bias voltages for (a) ITO/BPS-AN/Al, (b) ITO/TDP$\mathrm{AN} / \mathrm{Al}$, (c) Superposition conductance versus frequency for the two devices at $0 \mathrm{~V}$. 

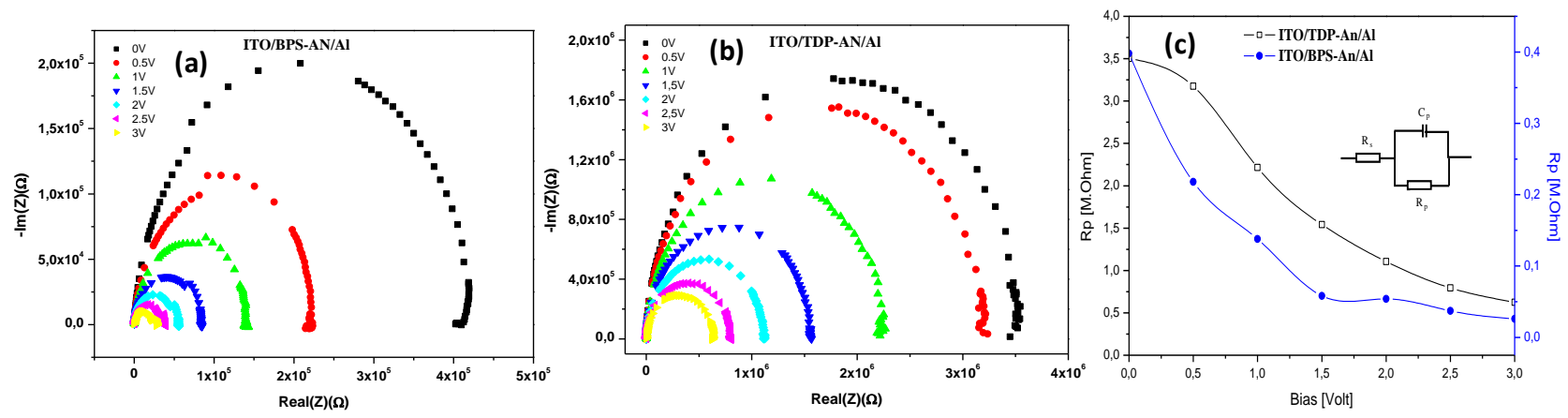

Figure 3. Cole-Cole plots of complex impedance with variation of the bias voltage for

(a) ITO/BPS-AN/Al, (b) ITO/TDP-AN/Al

(c) Superposition of the bulk resistance $R_{p}$ at bias voltage for the both structures

\section{Materials and Methods}

The anthracene-based organic materials were synthesized via the Williamson reaction through the condensation of $\mathrm{AnCl}$ (9-(Chloromethyl) anthracene) with two different bisphenols. Thus, the reaction of $\mathrm{AnCl}$ with BPS (4,4' Sulfonyldiphenol) led to BPS-AN whereas TDPAN was obtained from AnCl with TDP (4,4'thiodiphenol). These anthracene-based semiconducting materials were found to have good solubility in common organic solvents such as tetrahydrofuran, chloroform, DMF and DMSO.

Single-layer device was fabricated as sandwich structures between an aluminum ( $\mathrm{Al}$ ) cathode and an ITO anode (Fig 1.). ITO devices were cleaned for $20 \mathrm{~min}$ in an ultrasonic bath in acetone and isopropanol successively and finally dried by a nitrogen gas flow. After this treatment, a $50 \mu \mathrm{l}$ of an anthracene molecule was used to form a thin film on the ITO substrate by the spin-coating technique with a controlled speed of $2000 \mathrm{rpm}$. Finally, the obtained film was dried at $60{ }^{\circ} \mathrm{C}$ for 20 min to improve the adhesion between ITO and the anthracene layer.

Impedance spectroscopy (IS) measurements, a powerful technique for exploring the charge carrier mechanisms in materials and mainly in organic semiconductors, have been recorded using HP 4192 LF impedance analyzer.

\section{Conclusions}

Two anthracene derivatives were synthesized and presented in this work: TDP-AN and BPS-AN. Conductance measurements show the existence of two regimes: constant conduction at low-frequencies, and a power-law behaviour at high ones. Also, conductance results indicate that BPS-AN is more conductive than TDP-AN. Cole-Cole plots were modelled to extract the suitable equivalent circuit: resistance in series with capacitance and resistance in parallel. From the fitted parameters, it appears that the bulk resistance decreases with the applied bias, and that BPS-AN molecule is more conductive than the TDP-An one.

Conflicts of Interest: "The authors declare no conflicts of interest"

\section{References}

1. H. Haikel, H. Khaled, J. Nejmeddine, R.B. Chaabane, S. Olivier, M. Mustapha, New anthracene-based soluble polymers: optical and charge carrier transport properties, J Polym Res 2013, 20, 241-256.

2. H. Haikel, H. Khaled,M. Benzarti -Ghédira J. Nejmeddine, R.B. Chaabane, M. Mustapha, Optical and electrical characterization of thin films based on anthracene polyether polymers, Mater. Sci. Semicond. Process 2013, 16, 851-858.

3. I. Zahou, R. Ben Chaabane, R. Mlika, S. Touaiti, B. Jamoussi, H. Ben Ouada, Optical and electrical properties of novel peripherally tetra and mono -quinoleinoxy substituted metallophtalocyanines, J Mater Sci: Mater Electron 2014, 25, 2878-2888. 